University of Nebraska - Lincoln

DigitalCommons@University of Nebraska - Lincoln

\title{
Spatio-temporal Characteristics of Point and Field Sources in Wireless Sensor Networks
}

Mehmet C. Vuran

Georgia Institute of Technology, mcvuran@cse.unl.edu

Özgür B. Akan

Middle East Technical University

Follow this and additional works at: https://digitalcommons.unl.edu/cseconfwork

Part of the Computer Sciences Commons

Vuran, Mehmet C. and Akan, Özgür B., "Spatio-temporal Characteristics of Point and Field Sources in Wireless Sensor Networks" (2006). CSE Conference and Workshop Papers. 148.

https://digitalcommons.unl.edu/cseconfwork/148

This Article is brought to you for free and open access by the Computer Science and Engineering, Department of at DigitalCommons@University of Nebraska - Lincoln. It has been accepted for inclusion in CSE Conference and Workshop Papers by an authorized administrator of DigitalCommons@University of Nebraska - Lincoln. 


\section{Spatio-temporal Characteristics of Point and Field Sources in Wireless Sensor Networks}

\author{
Mehmet C. Vuran \\ Broadband \& Wireless Networking Laboratory \\ School of Electrical \& Computer Engineering \\ Georgia Institute of Technology, Atlanta, GA 30332 \\ Email: mcvuran@ece.gatech.edu
}

\author{
Özgür B. Akan \\ Department of Electrical and Electronics Engineering \\ Middle East Technical University \\ 06531, Ankara, Turkey \\ Email: akan@eee.metu.edu.tr
}

\begin{abstract}
Wireless Sensor Networks (WSN) are comprised of densely deployed sensor nodes collaboratively observing and communicating extracted information about a physical phenomenon. Dense deployment of sensor nodes makes the sensor observations highly correlated in the space domain. In addition, consecutive samples obtained by a sensor node are also temporally correlated for the applications involving the observation of the variation of a physical phenomenon. Based on the physical characteristics and dispersion pattern over the area, the phenomenon to be observed can be modeled as point source or field source. Clearly, understanding the spatio-temporal correlation characteristics of the point and field sources brings potential advantages to be exploited in the design of efficient communication protocols. In this paper, a theoretical analysis of spatio-temporal correlation in WSN is carried out. The objective of this analysis is to capture the spatio-temporal characteristics of point and field sources in WSN. First, the model for point and field sources are developed and their spatio-temporal characteristics are analytically derived along with the distortion functions. Based on the theoretical analysis, numerical simulations are performed. This analytical work provides tools for finding the feasible operating region in terms of spatial and temporal resolution for a specific distortion constraint considering spatio-temporal correlation, signal properties, and network variables in WSN.
\end{abstract}

Index Terms-Spatio-temporal correlation, point source, field source, distortion, wireless sensor networks.

\section{INTRODUCTION}

W IRELESS Sensor Networks (WSN) are generally comprised of densely deployed sensor nodes collaboratively observing and communicating extracted information about physical phenomenon [1]. In general, accurate and efficient operation of any WSN deployment necessitates to maintain sufficient network and sensing coverage in the sensor network. To assure network and sensing coverage, WSN applications require sensor nodes to be densely deployed in the field. Dense deployment of sensor nodes makes the sensor observations highly correlated in the space domain with the degree of correlation increasing with internode proximity. Similarly, some of WSN applications such as event tracking require sensor nodes to periodically sample and communicate the sensed event features. The nature of the energy-radiating physical phenomenon yields temporal correlation between

The work of Mehmet C. Vuran is supported by the National Science Foundation under contract CNS-0519841 and the work of Özgür B. Akan is supported by the Turkish Scientific and Technical Research Council (TUBITAK) Career Award under grant KARIYER-104E043. each consecutive observation of a sensor node [8]. The degree of correlation between consecutive sensor measurements may vary according to the temporal variation characteristics of the phenomenon.

Based on the application, the physical phenomenon to be observed can be modeled as point source, e.g., target detection/tracking, or field source, e.g., monitoring of magnetic field and seismic activities. In general, events generating signal which originates from a single point in the field can be modeled as a point source. The cases, where the physical phenomenon is dispersed over the field, can be modeled as field source. Clearly, it is of great importance to capture the spatio-temporal characteristics of point and field sources to be able design energy-efficient communication protocols which can exploit the potential advantages of correlation in WSN.

There has been some research efforts to study the correlation in WSN [2]-[7]. In [3], the problem of correlated data gathering with the objective of energy minimization is studied using different coding models. Similar analysis is performed in [4] for lossy network and high-resolution coding under distortion constraints. The authors of [5] investigate the optimal network density under the total distortion constraint for delay-sensitive real-time data gathering of correlated physical phenomenon in WSN. Similarly, in [6], the joint optimization of sensor placement and transmission structure for data gathering is proposed. However, these studies consider only the spatial correlation in the WSN and do not incorporate the temporal correlation in their analysis. In [7], a lower bound on the best achievable end-to-end distortion for different coding schemes is derived as a function of the network parameters. Moreover, in [2], the relation between spatial and temporal sampling rate on the overall network delay and energy consumption is studied. However, these work neither consider nor capture the spatiotemporal characteristics of the physical phenomenon. In [8], the individual spatial and temporal correlation characteristics of a field source is considered. However, this work does not consider the joint effects of spatio-temporal correlation for both point and field sources. Hence, despite the considerable amount of existing research on correlation in WSN, none of these works explicitly study the spatio-temporal characteristics of physical phenomenon modeled by point and field sources.

In this paper, a theoretical analysis of spatio-temporal correlation in WSN is carried out. The objective of this 
analysis is to capture the spatio-temporal characteristics of physical phenomenon modeled by point and field sources in WSN. This work extends [8] by considering joint effects of spatio-temporal correlation for both point and field sources. In this study, first, the model for point and field sources are introduced and their spatio-temporal characteristics are derived along with the distortion functions in Section II and Section III, respectively. Based on the theoretical analysis, in Section IV, the numerical simulations for spatio-temporal correlation characteristics of point and field sources are performed. The results of this study yield a significant theoretical background to be used in the development of efficient communication protocols. Finally, the concluding remarks are discussed in Section V.

\section{Point Source}

In many WSN applications such as target detection and fire detection, the goal is to estimate the properties of an event generated by a single point source, through collective observations of sensor nodes. In this section, we first introduce our model for the point source and formulate its spatio-temporal characteristics. Next, we derive the distortion function for the estimation of the point source.

\section{A. Spatio-temporal Characteristics}

The point source is assumed to generate a continuous signal which is modeled by a random process $f_{S}(s, t)$, where $s$ denotes the outcome and $t$ denotes time. For ease of illustration, we use $f_{S}(t)$ in the remaining of the paper. We model the point source, $f_{S}(t)$, as a gaussian random process such that $f_{S}(t)$ is first-order stationary, i.e., $\mu_{S}(t)=\mu_{S}$ and has a variance $\sigma_{S}^{2}$. Without loss of generality, we assume $\mu_{S}=0$.

For ease of illustration, we assume the coordinate axis is centered at the point source. As a result, the received signal, $f(x, y, t)$, at time $t$ at a location $(x, y)$ can be modeled as

$$
f(x, y, t)=f_{S}\left(t-\frac{\sqrt{x^{2}+y^{2}}}{v}\right) e^{-\frac{\sqrt{x^{2}+y^{2}}}{\theta_{s}}},
$$

which is the delayed and attenuated version of the signal $f_{S}(t)$. In this model, we assume that the event signal travels with the speed, $v$, and is attenuated based on an exponential law, where $\theta_{s}$ is the attenuation constant. Note that, the function $f(x, y, t)$ is also a Gaussian random process and the samples taken by the sensors are jointly Gaussian random variables (JGRVs). Since, $\mu_{S}=0$, the mean of the received signal, $\mu_{E}=0^{1}$. The variance of the received signal is also given as follows:

$$
\sigma_{E}^{2}(x, y)=E\left[f^{2}(x, y, t)\right]=\left(\sigma_{S} e^{-\sqrt{x^{2}+y^{2}} / \theta_{s}}\right)^{2} .
$$

An interesting result from (2) is that, the variance of the signal observed at location $(x, y)$ depends on the distance between the observation location and the point source. The received signal at time $t_{k}$ by a sensor $n_{i}$ at location $\left(x_{i}, y_{i}\right)$ is given by

$$
S_{i}[k]=f\left(x_{i}, y_{i}, t_{k}\right) .
$$

\footnotetext{
${ }^{1}$ The subscripts $S$ and $E$ which are used throughout the paper represent the source and event, respectively.
}

Assuming wide-sense stationarity, the spatio-temporal correlation function for two samples of a point source taken at locations $\left(x_{i}, y_{i}\right)$ and $\left(x_{j}, y_{j}\right)$, and at times $t_{k}$ and $t_{l}$, respectively, is given by

$$
\begin{aligned}
\rho_{p}(i, j, k, l) & =\frac{E\left[S_{i}[k] S_{j}[l]\right]}{\sigma_{E}\left(x_{i}, y_{i}\right) \sigma_{E}\left(x_{j}, y_{j}\right)} \\
& =\rho_{S}\left(\Delta_{t}\right),
\end{aligned}
$$

where $\Delta_{t}=\left|t_{k}-t_{l}-\left(d_{i}-d_{j}\right) / v\right|, d_{i}=\sqrt{x_{i}^{2}+y_{i}^{2}}$ is the distance of the sensor $n_{i}$ to the point source, and $\rho_{S}\left(\Delta_{t}\right)=$ $E\left[f_{S}(t) f_{S}\left(t+\Delta_{t}\right)\right] / \sigma_{S}^{2}$ is the correlation function of the point source which is given by $\rho_{S}\left(\Delta_{t}\right)=e^{-\Delta_{t} / \theta_{t}}$, where $\theta_{t}$ is a constant governing the degree of correlation. Note that the spatio-temporal correlation between two samples, $\rho_{p}(i, j, k, l)$, depends mainly on the difference between sample times $t_{k}$ and $t_{l}$ since generally $v \gg\left(d_{i}-d_{j}\right)$.

\section{B. Distortion in WSN}

In WSN, we are interested in estimating the signal generated by the point source using the samples collected by the sensor nodes. The expectation of the generated signal, $f_{S}(t)$, over an interval $\tau$ is given by

$$
S(\tau)=\frac{1}{\tau} \int_{0}^{\tau} f_{S}(t) d t
$$

Each sensor node, $n_{i}$, receives the attenuated and delayed version of the generated signal $f_{S}(t)$, i.e., $S_{i}[k]$. Due to the impurities in the sensor circuitries, the sampled signal is the noisy version of this received signal which is given by

$$
X_{i}[k]=S_{i}[k]+N_{i}[k],
$$

where the subscript $i$ denotes the location of the node $n_{i}$, i.e., $\left(x_{i}, y_{i}\right), k$ denotes the sample index which corresponds to time $t=t_{k}{ }^{2}, X_{i}[k]$ is the noisy version of the actual sample $S_{i}[k]$, and $N_{i}[k]$ is the observation noise, i.e., $N_{i}[k] \sim \mathcal{N}\left(0, \sigma_{N}^{2}\right)$. $S_{i}[k]$ is given by (1) and (3).

The observed information, $X_{i}[k]$, is then encoded and sent to the sink through the WSN. It has been shown that joint source-channel coding outperforms separate coding. Moreover, for WSN with finite number of nodes, uncoded transmission outperforms any approach based on the separation paradigm leading to the optimal solution for infinite number of nodes [8]. Under the light of these results, we assume that uncoded transmission is deployed in each node. Hence, the transmitted observation, $Y_{i}[k]$, is given by

$$
Y_{i}[k]=\sqrt{\frac{P_{E}}{\sigma_{S}^{2}+\sigma_{N}^{2}}} X_{i}[k], \quad i=1, \ldots, N
$$

where $\sigma_{S}^{2}$ and $\sigma_{N}^{2}$ are the variances of the event information $S_{i}[k]$ and the observation noise $N_{i}[k]$, respectively.

The transmitted information is decoded at the sink. Since uncoded transmission is used, it is well known that minimum

\footnotetext{
${ }^{2}$ Note that we use a discrete-time model since each node is assumed to sample the physical phenomenon synchronously after the initial wake-up.
} 


$$
\begin{aligned}
D_{p}(\tau, f, M)= & \sigma_{S}^{2}-\frac{2}{\tau^{2} f M} \sum_{i=1}^{M} \sum_{k=1}^{\tau f} \frac{\sigma_{S}^{4} e^{-3 d_{i} / \theta_{s}}}{\sigma_{S}^{2} e^{-2 d_{i} / \theta_{s}}+\sigma_{N}^{2}} \theta_{t}\left[2-e^{-\left(t_{k}+d_{i} / c\right)}-e^{-\left(\tau-t_{k}-d_{i} / c\right) / \theta_{t}}\right] \\
& +\frac{\sigma_{N}^{2}}{\tau f M^{2}} \sum_{i=1}^{M} \frac{\sigma_{S}^{4} e^{-2 d_{i} / \theta_{s}}}{\left(\sigma_{S}^{2} e^{-d_{i} / \theta_{s}}+\sigma_{N}^{2}\right)^{2}}+\frac{1}{\tau^{2} f^{2} M^{2}} \sum_{i=1}^{M} \sum_{j=1}^{M} \sum_{k=1}^{\tau f} \sum_{l=1}^{\tau f} \alpha \rho(i, j, k, l),
\end{aligned}
$$

mean square error (MMSE) estimation is the optimum decoding technique [8]. Hence, the estimation, $Z_{i}[k]$, of the event information $S_{i}[k]$ is simply the MMSE estimation of $Y_{i}[k]$, which is given by

$$
Z_{i}[k]=\frac{\sigma_{E}^{2}\left(x_{i}, y_{i}\right)}{\sigma_{E}^{2}\left(x_{i}, y_{i}\right)+\sigma_{N}^{2}}\left(S_{i}[k]+N_{i}[k]\right)
$$

The sink is interested in estimating the expected value of the event during a decision interval $\tau$ which is given by (5). Assuming each sensor node sends information at a rate of $f$ samples/sec, this estimation can simply be found by

$$
\hat{S}(\tau, f, M)=\frac{1}{\tau f M} \sum_{i=1}^{M} \sum_{k=1}^{\tau f} Z_{i}[k]
$$

where $M$ is the number of sensor nodes that send samples of the observed point source. $M$ nodes are chosen among the nodes in the network to represent the point source, and hence, are referred to as representative nodes. Consequently, the distortion achieved by this estimation is given by

$$
D_{p}(\tau, f, M)=E\left[(S(\tau)-\hat{S}(\tau, f, M))^{2}\right]
$$

where the subscript $p$ denotes the point source. Using (1), (2), (5), (8), and (9), (10) can be expressed as in (11), where

$$
\alpha=\frac{\sigma_{S}^{8} e^{-2\left(d_{i}+d_{j}\right) / \theta_{s}}}{\left(\sigma_{S}^{2} e^{-d_{i} / \theta_{s}}+\sigma_{N}^{2}\right)\left(\sigma_{S}^{2} e^{-d_{j} / \theta_{s}}+\sigma_{N}^{2}\right)},
$$

$d_{i}=\sqrt{\left(x_{i}+y_{i}\right)}$, and $\rho(i, j, k, l)$ is the spatio-temporal correlation function given in (4).

\section{FIELD SOURCE}

There exist applications such as temperature monitoring and seismic monitoring, where the physical phenomenon is dispersed over the sensor field, and, hence, can be modeled as a field source. Thus, here, we explore the spatio-temporal characteristics of observing such a phenomenon in WSNs.

\section{A. Spatio-temporal Characteristics}

The event signal $f(x, y, t)$ is assumed to be a Gaussian random process with $\mathcal{N}\left(0, \sigma_{s}^{2}\right)$. The sink is interested in estimating the signal $f\left(x_{0}, y_{0}, t\right)$ over the decision interval $\tau$ at location $\left(x_{0}, y_{0}\right)$. Assuming the observed signal $f(x, y, t)$ is wide-sense stationary (WSS), the expectation of the signal over the decision interval $\tau$, i.e., $S(\tau)$ can be calculated by the time average of the observed signal:

$$
S(\tau)=\frac{1}{\tau} \int_{0}^{\tau} f\left(x_{0}, y_{0}, t\right) d t
$$

where $\left(x_{0}, y_{0}\right)$ is the event location. The signal, $S_{i}[k]$ received at time $t_{k}$ by a sensor node at location $\left(x_{i}, y_{i}\right)$ is defined as in (3) and $S_{i}[k]$ 's are JGRV with $\mathcal{N}\left(0, \sigma_{s}^{2}\right)$. The covariance of two samples, $S_{i}[k]$ and $S_{j}[l]$, is given by:

$$
\operatorname{cov}\left\{S_{i}[k], S_{j}[l]\right\}=\sigma_{S}^{2} \rho_{s}(i, j) \rho_{t}(\delta),
$$

where

$$
\rho_{s}(i, j)=e^{-d_{i, j} / \theta_{s}}, \text { and } \rho_{t}(\delta)=e^{-|\delta| / \theta_{t}}
$$

are spatial and temporal correlation functions, respectively, $\delta=(k-l) / f, f$ is the sampling rate, $d_{i, j}=$ $\sqrt{\left(x_{i}-x_{j}\right)^{2}+\left(y_{i}-y_{j}\right)^{2}}$ is the distance between two nodes $n_{i}$ and $n_{j}$, and $\theta_{s}$ and $\theta_{t}$ are spatial and temporal correlation coefficients, respectively.

\section{B. Distortion in WSN}

Following the discussion and derivations in Section II, the noisy version of the signal, $X_{i}[k]$, the transmitted signal, $Y_{i}[k]$ are given by (6) and (7), respectively. The estimation $Z_{i}[k]$ can be found as follows:

$$
Z_{i}[k]=\frac{\sigma_{S}^{2}}{\sigma_{S}^{2}+\sigma_{N}^{2}}\left(S_{i}[k]+N_{i}[k]\right) .
$$

After collecting the samples of the signal in the decision interval $\tau$ from $M$ nodes, the sink estimates the expectation of the signal over the last decision interval as given in (9). As a result, the distortion achieved by this estimation is given as in (10). Using the definitions above and substituting (12), (15), and (9) into (10); the distortion function can be derived as in (16).

\section{ANALYSIS AND RESUlts}

In this section, we provide numerical simulation results for spatio-temporal correlation characteristics of point and field sources using the distortion functions given by (11) and (16). A sensor network of a grid topology of $50 \mathrm{~m} \times 50 \mathrm{~m}$ with 120 nodes is used for the evaluations. For the point source evaluations, the location of the point source is the center of the grid, while for the field source evaluations, the network aims to estimate the signal value at the center of the grid using the samples of the sensors located on the grid.

For each evaluation, the closest $M$ nodes to the center are chosen to send information. This selection method, which we refer to as ordered selection, enables us to observe the spatial correlation effects as the $M$ value is increased, since higher value of $M$ corresponds to nodes farther from the center being chosen. Another important parameter is the number of packets sent by a single sensor node during the decision interval $\tau$, 


$$
\begin{aligned}
D_{f}(\tau, f, M)= & \sigma_{S}^{2}-\frac{2 \sigma_{S}^{2}}{\tau^{2} f M\left(\sigma_{S}^{2}+\sigma_{N}^{2}\right)} \sum_{i=1}^{M} \rho_{s}(i, s) \sum_{k=1}^{\tau f} \theta_{t}\left[2-e^{-k /\left(f \theta_{t}\right)}-e^{-\left(t-\frac{k}{f}\right) / \theta_{t}}\right] \\
& +\frac{\sigma_{S}^{4} \sigma_{N}^{2}}{\tau f M\left(\sigma_{S}^{2}+\sigma_{N}^{2}\right)^{2}}+\frac{\sigma_{S}^{6}}{\left(\tau f M\left(\sigma_{S}^{2}+\sigma_{N}^{2}\right)\right)^{2}} \sum_{i=1}^{M} \sum_{j=1}^{M} \sum_{k=1}^{\tau f} \sum_{l=1}^{\tau f} \rho_{s}(i, j) \rho_{t}(|k-l| / f)
\end{aligned}
$$

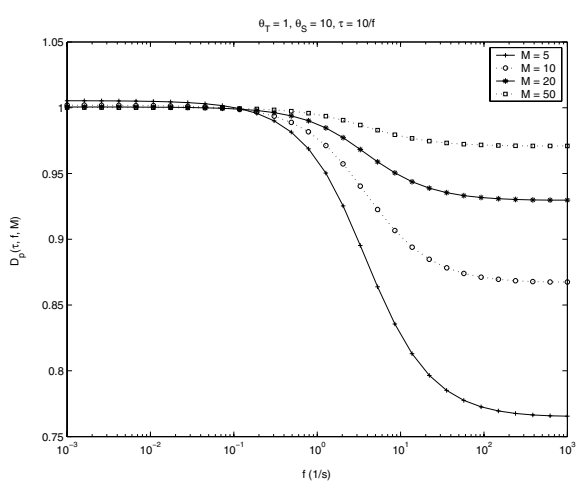

(a)

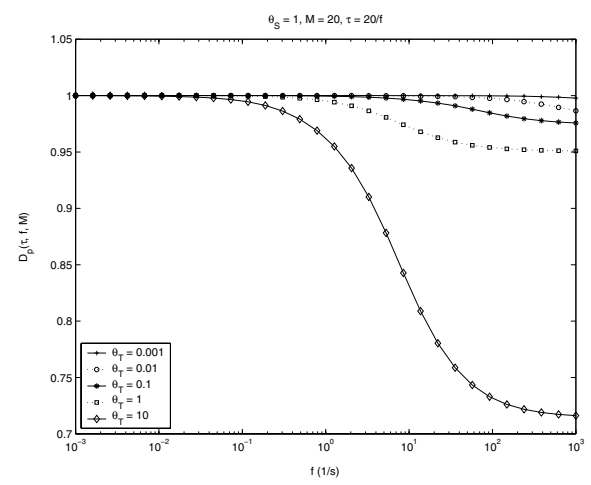

(c)

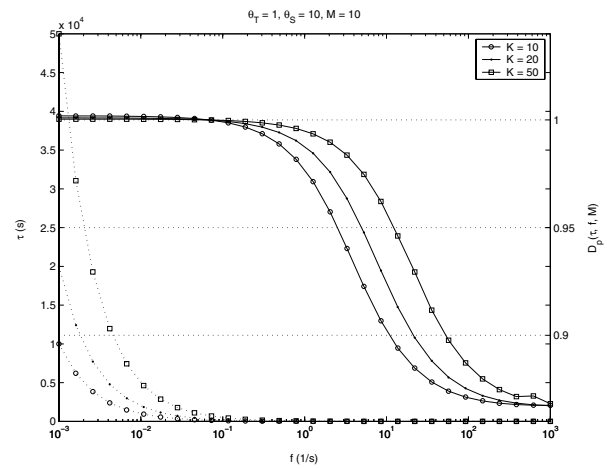

(b)

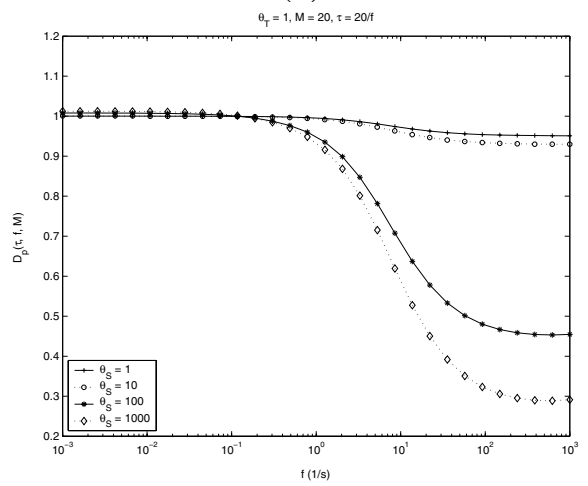

(d)

Fig. 1. Distortion vs. sampling rate for different values of (a) $M$, (b) $K$, (c) $\theta_{T}$, and (d) $\theta_{S}$ (Point source).

which is denoted as $K=\tau f$, where $f$ is the sampling rate of the sensor nodes. In the evaluations, the parameter $K$ is fixed and $\tau$ is determined as the $f$ changes, which enables us to investigate the effect of a large range of sampling rate values.

\section{A. Point Source}

In this section, the behavior of the distortion function in (11) is investigated for various values for sampling rate, $f$, number of representative nodes, $M$, number of samples, $K$, and spatial and temporal correlation coefficients, $\theta_{S}$ and $\theta_{T}$, respectively. The influence of sampling rate, $f$, on distortion is shown in Fig. 1(a). It is clearly seen that, as the sampling rate increases, distortion decreases, which show the effect of temporal resolution on event estimation. The rate of decrease is significantly large for a specific range of $f$ values, e.g., $0.1<f<100$ for $M=5$. Moreover, above this range of $f$ values, the distortion remains relatively constant. This observation reveals that there is an optimal value, $f_{\text {opt }}$, for temporal resolution such that further increase in sampling rate, $f$, does not influence the distortion.
The effect of number of representative nodes, $M$, on distortion is also shown in Fig. 1(a). It is clear that, increasing $M$ degrades the distortion function for high values of sampling rate, $f$. As the sampling rate increases, consecutive samples become sufficient to extract the characteristics of the source. However, as $M$ is increased, distortion increases due to decrease in spatial correlation. On the other hand, for lower sampling rates, e.g., $f<0.1 s^{-1}$, an increase in $M$ improves the distortion since the temporal resolution is not sufficient in this case. As a result of increased $M$, the spatial correlation helps build a more accurate estimation of the signal. However, increasing $M$ above a specific value, e.g., $M=10$, has no impact on distortion. This result reveals that, there is an optimal value, $M_{o p t}$, for efficient estimation, which we will investigate in detail later.

In Fig. 1(b), the effect of number of samples, $K$, is shown. The solid lines represent the distortion values for each $K$, and the dotted lines show the corresponding decision interval values, i.e., $\tau$. Note that, when the sampling rate is low, $\tau$ 


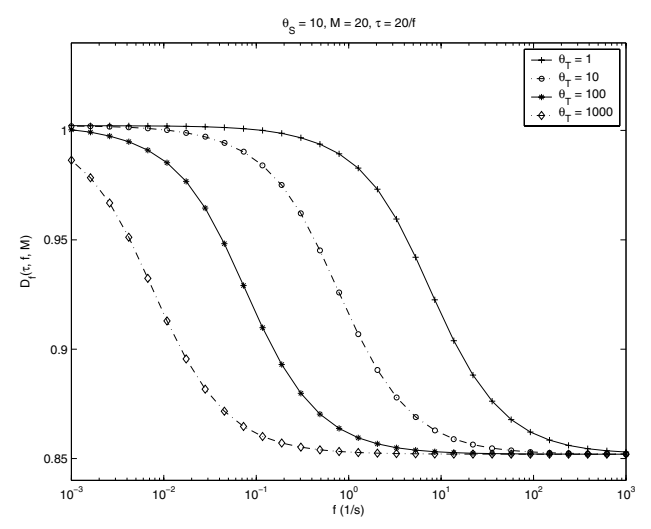

Fig. 2. Distortion vs. sampling rate for different values of $\theta_{T}$ (Field source).

increases significantly so that required number of samples can be collected (note the logarithmic scale on $x$-axis). However, this increase in $\tau$ results in temporally uncorrelated samples to be collected, which leads to higher distortion. However, for higher values of $f, \tau$ decreases and the temporal resolution becomes sufficient. Moreover, for the transition region, where the distortion function decreases significantly, the number of samples, $K$, has an important influence on distortion. In this region, lower $K$ also improves the estimation since more closely sampled instances are taken into account. However, when the sampling rate, $f$, is further increased, the temporal resolution becomes so fine that, any number of samples does not effect the distortion, where the lines for different $K$ values intersect.

In Fig. 1(c) and 1(d), the influence of the temporal and spatial correlation coefficients, $\theta_{T}$ and $\theta_{S}$, on distortion are shown, respectively. A higher value of either of these parameters corresponds to a higher correlation in either temporal or spatial domain. As shown in Fig. 1(c), as $\theta_{T}$ is increased, i.e., the temporal correlation between nodes are increased, an increase in sampling rate results in a much more decrease in the distortion. This is also observed for $\theta_{S}$ as shown in Fig. 1(d). An interesting result is that, although the number of nodes are fixed in Fig. 1(d), higher spatial correlation improves the effect of temporal resolution since each selected node is highly correlated with the point source.

\section{B. Field Source}

In this section, the behavior of the distortion function in (16) for field sources is investigated for the same set of parameters used in Section IV-A. The effect of the number of representative nodes, $M$, and the number of samples, $K$ are found to be similar to the point source case and hence, are not reproduced here. Similar observations can be made as in Section IV-A.

In Fig. 2, the effect of temporal correlation coefficient on the distortion is shown. Contrary to our results for point source, the decrease in distortion does not depend on $\theta_{T}$. However, the distortion plot shifts to the left when $\theta_{T}$ is increased. This

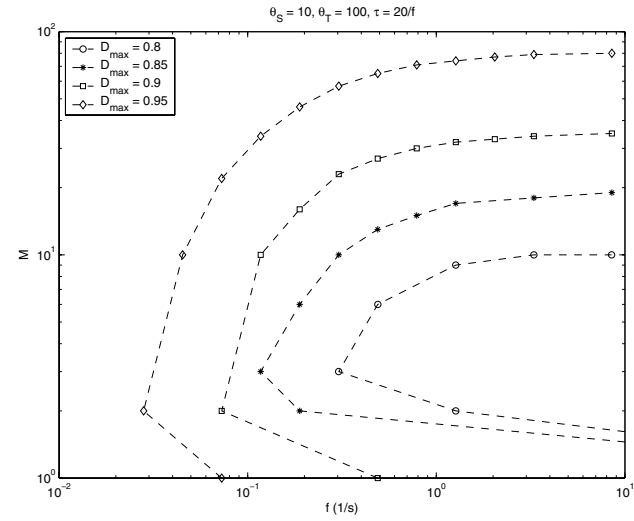

Fig. 3. Number of nodes vs. sampling rate, $(M, f)$ tuples meeting various $D_{\max }$ constraints (Field source).

affects the optimal sampling rate value, $f_{o p t}$, i.e., higher $\theta_{T}$ results in smaller $f_{o p t}$ value. On the other hand, the relation between spatial correlation coefficient $\theta_{S}$ with distortion is similar to the case in point source shown in Fig. 1 (d), and hence, is not reproduced here. Similarly, the decrease in distortion depends on $\theta_{S}$, which shows that spatial correlation is more important in decreasing the distortion for field sources.

In Fig. 3, the tradeoff between spatial and temporal resolution is shown. Each point represents the boundary of the feasible region for $(M, f)$ values that meet a certain distortion constraint, $D_{\max }$. The figure can be read as follows: For each allowed distortion $D_{\max }$, the tuples represent the boundary of the feasible region inside which the distortion constraint is guaranteed. An important result is that, for each $D_{\max }$ value, there is an optimum operating point, where minimum number of nodes can be used with low sampling rate. Increasing $M$ above this value also requires increase in temporal resolution. Moreover, a decrease in maximum allowed distortion value, $D_{\max }$, results in a smaller feasible region, as expected. This also results in a smaller range for feasible values of $M$. Consequently, aggressively collecting information from each sensor node in the field, does not necessarily correspond to more accurate estimation. This figure serves as an important guideline to the design of communication protocols, network topology and deployment for a particular distortion requirement.

\section{External Parameters}

In this section, we investigate the effects of the network topology properties, such as grid size, and the effects of using a random topology for field sources. The results also apply to point sources and are not shown here because of space limitations.

We present the effects of network topology on event distortion in Fig. 4, where distortion is plotted versus $M$ for different grid sizes at sampling rate $f=13.74 \mathrm{~s}^{-1}$. Grid size is defined as the minimum distance between two neighbor nodes in the grid topology. Smaller grid sizes correspond to higher granularity at the cost of higher density and larger number of 


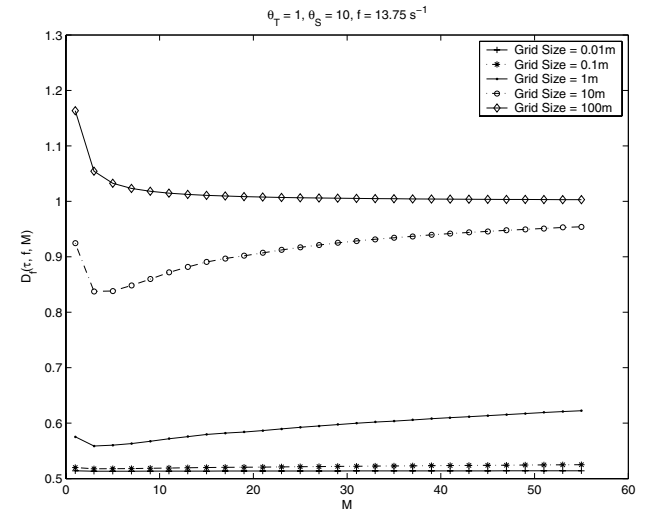

Fig. 4. Distortion vs. $M$ for different grid sizes, $f=13.74$ (Field source).

sensors to cover a certain area, which affect the deployment cost and energy consumption of the WSN. It is shown in Fig. 4 that there is an optimal point for $M$ for small grid sizes $(<10$ $\mathrm{m})$. Moreover, increasing grid size also increases the distortion. As explained before, when $M$ is increased, nodes far from the field center are chosen. Hence, as the grid size increases, these nodes become spatially uncorrelated. In this case, increasing $M$ helps decrease distortion. On the other hand, when the grid size is small, an increase in $M$ does not affect the distortion. This shows that a suitable internode distance needs to be chosen for efficient coverage of the physical phenomenon as well as an optimum $M$ value.

So far, a grid topology has been considered for evaluations. However, a grid topology may not be practical for some WSNs and the effect of using a random topology needs to be considered. Moreover, in our evaluations, we use a specific method for representative node selection, such that the closest $M$ nodes to the source are chosen. This selection assumes that the location of the event source is known. However, in some applications, the location of the source may not be available to the sink. This is important especially in tracking applications.

The effect of randomness in the network topology and the node selection method is investigated using three different scenarios. The first scenario corresponds to a random topology of 120 nodes in a $50 \mathrm{~m} \times 50 \mathrm{~m}$ topology, where $M$ representative nodes are randomly selected. For Scenario 2, again random topology is used with ordered selection method. Scenario 3 corresponds to grid topology with ordered selection. The achieved distortion from these scenarios are shown in Fig. 5. For scenarios 1 and 2, the average of 1000 random topologies are shown. The results show that, when ordered selection is used, the randomness of the network topology improves the achieved distortion. It can also be observed that, when the nodes are randomly chosen, the achieved distortion is significantly high, i.e., Scenario 1, since the representative nodes can be located anywhere in the network. However, a higher value of $M$, in this case, helps reduce the distortion. Hence, in practical applications where the source location is not known, at first, higher number of representative nodes can be used to locate the signal source, and once the location

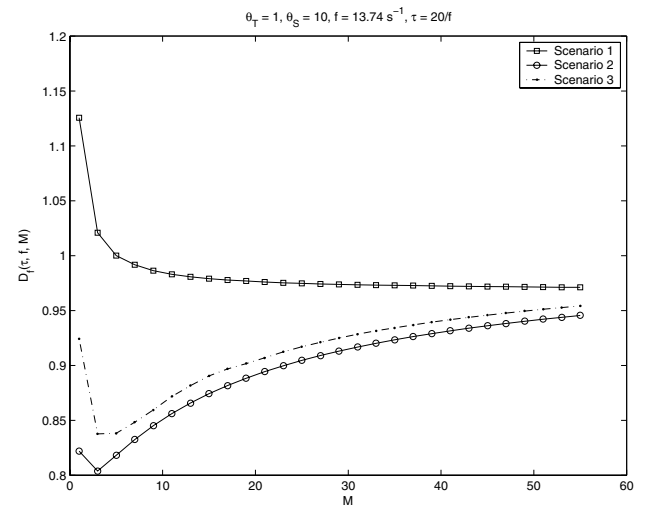

Fig. 5. Distortion vs. $M$ for different network topology and node selection procedures (Field source).

of the signal source is found, the number of nodes can be decreased to the optimum $M$ value to improve the accuracy of estimation.

\section{CONCLUSION}

In this paper, a theoretical analysis of spatio-temporal correlation characteristics of point and field sources in WSN is performed. This analytical work provides tools for finding the feasible operating region in terms of spatial and temporal resolution for a specific distortion constraint considering spatio-temporal correlation, signal properties, and network variables in WSN. Extensions of this work include the study of spatio-temporal characteristics of point and field sources with different correlation models. Moreover, we will investigate different reconstruction models such as filtering or nearest node representation. Furthermore, the effect of the impurities of the wireless channel will be investigated. Finally, the comparison of the correlation models with empirical data will be conducted.

\section{REFERENCES}

[1] I. F. Akyildiz, W. Su, Y. Sankarasubramaniam, and E. Cayirci, "Wireless Sensor Networks: A Survey," Computer Networks (Elsevier) Journal, vol. 38, no. 4, pp. 393-422, March 2002.

[2] S. Bandyopadhyay and E. J. Coyle, "Spatio-temporal sampling rates and energy efficiency in wireless sensor networks," in Proc. IEEE INFOCOM 2004, vol. 3, pp. 1728-1739, 2004.

[3] R. Cristescu, B. Beferull-Lozano, and M. Vetterli, "On network correlated data gathering," in Proc. IEEE INFOCOM 2004, vol. 4, pp. 2571-2582, March 2004.

[4] R. Cristescu and B. Beferull-Lozano, "Lossy network correlated data gathering with high-resolution coding," in Proc. IPSN 2005, pp. 218-224, April 2005.

[5] R. Cristescu and M. Vetterli, "On the optimal density for real-time data gathering of spatio-temporal processes in sensor networks", in Proc. IPSN 2005, April 2005.

[6] D. Ganesan, R. Cristescu, B. Beferull-Lozano, "Power-efficient sensor placement and transmission structure for data gathering under distortion constraints," in Proc. IPSN 2004, pp. 142 - 150, April 2004.

[7] M. Gastpar, M. Vetterli, "Power, spatio-temporal bandwidth, and distortion in large sensor networks," IEEE Journal on Selected Areas in Communications, vol. 23, no. 4, pp. 745 - 754, April 2005.

[8] M. C. Vuran, O. B. Akan, and I. F. Akyildiz, "Spatio-Temporal Correlation: Theory and Applications for Wireless Sensor Networks," Computer Networks Journal (Elsevier), vol. 45, no. 3, pp. 245-261, June 2004. 